\title{
13
}

\section{Corruption as a social process from dyads to networks}

\author{
John Warburton
}

The problem of corruption can be stated in relatively simple terms. What is it? How does it function? If we could answer these questions we should be able to discover ways of combating what is now perceived as the scourge of the modern world. However, every complex problem has a simple plausible answer, which is invariably wrong. The problem of corruption may be able to be framed in uncomplicated terms but this is where the simplicity ends.

In general terms, corruption is an artefact of social and political organisation and, as such, is a phenomenon of infinite complexity. I have never seen a definition or paradigm that comprehensively describes corruption in all its guises and manifestations, nor do I propose one here. In this chapter I will argue that understanding corruption as a social process has implications and advantages that provide insights into the way corruption functions. I will be suggesting that such insights can also inform corruption prevention programs.

The orthodox way to approach corruption these days is to examine the institutions and systems that allow corruption to occur. To some extent I am turning this on its head by suggesting that if we are to understand corruption as a social process then we need to understand the individual actors who are the participants in this social system. I'm not suggesting that the institutional approach is wrong but we are attacking the same problem from somewhat different directions. Larmour and Wolanin are right to point out, in the introduction to this volume, that the managerialist approach to corruption invariably fails because of a failure to address the atmosphere of integrity surrounding the institution. I would add that this approach fails to incorporate the cognition, motivations and actions of individuals. Addressing the individual seems to be out of fashion because it reminds some of the unsophisticated 'rotten apple theory' that was usually trotted out to explain corruption in simpler times. 
We have now moved full circle with several contributors in this volume citing Zipparo's (1998) argument that deviance stems largely from the nature of the organisation rather than the nature of the individual. Instead, I would suggest that to come to some fundamental understanding of the way corruption functions it is essential to understand it firstly from the individual's perspective and to then build the individual into a complex social world. This has not been done to date, probably because it is such a difficult and complex task, requiring an interdisciplinary approach. In any case I will attempting to propose a different paradigm describing the way corrupt transactions occur and the way corrupt networks develop.

\section{Corruption as a social process}

Corrupt transactions occur between actors as the result of social interaction. For corrupt transactions to occur there must be communication between two or more individuals. Individuals intend to participate in corrupt transactions. They may not call them corrupt or they may have some rationalisation to hand to justify their behaviour, but they intend to promote their interests over the interests of others. The precise nature of those interests and how actors come to have them is not my concern here. Actors have interests/goals/desires/needs/preferences ${ }^{1}$ and they act to achieve them. ${ }^{2}$ The most important factor is not the nature of the interests but the strength of the actor's desire to achieve them. Petri refers to the 'energy' inherent in motivation that drives actors to act (1981:18).

Promoting your own interest is essentially identifying and attempting to realise personal goals. In order for an actor to achieve her goals she must have 'power' (Lamberth 1980:295). It is my contention that both parties to corrupt transactions are resource holders and power seekers. They possess differing resources of power that cannot easily be converted to promote their own interests. The purpose of corrupt transactions is to conduct a swap, which converts resources into usable power. The participants in the transaction can then use the power they have obtained from the transaction to promote their own interest.

In any such transaction each individual actor has to make a personal decision as to whether they will instigate or accede to an attempted corrupt transaction. If the actor participates in such a transaction then it can be assumed they have crossed a decisional threshold. I contend there are two competing forces acting on them as they reach the decisional threshold. Firstly, there are motivational, or excitatory forces, energising them toward participation in the corrupt transaction. Principally, they are fuelled by the strength of the actor's desire to promote the interest that the corrupt transaction 
will satisfy. This strength of desire will be a function of the actor's dependence on the resource that the other actor in the transaction controls and is offering as part of the transaction. ${ }^{3}$ Secondly, there are inhibitory forces lowering the tendency of the actor to cross the decisional threshold. These would include such factors as moral inhibition, perception of the risk of sanction and perception of the consequences of sanction.

It becomes obvious from this discussion that the systemic effects of organisational culture are only one of the factors that affect the individual at the decisional threshold. It is true that organisational culture and systems change can affect the scale or opportunity for corrupt transactions. Such factors address the inhibitory forces acting on an individual actor's decision to behave corruptly. They do not however address the motivational forces impelling an actor to behave corruptly. I would like to address these forces and examine how they can lead to the corrupt transactions and the development of corrupt networks of exchange.

\section{Corruption and power}

The connection between corruption and power has been poorly understood to date, with few theorists explicitly drawing a connection. Rogow and Lasswell's Power, Corruption and Rectitude is one of the few works to link corruption and power but sheds little real light on the connection (1963). It concentrates on power in its broadest political sense and failed to place it in the context of individual transactions.

It is my contention that corrupt transactions are a special form of power relations that involve a mutual swapping of the resources of power. The precise nature of power is contentious and I will only say here that I will draw on the Weberian causal concept of power, ${ }^{4}$ combined with Emerson's theory of resources dependency. ${ }^{5}$ My view is that power is a generic term used to describe the state of being of an actor, ${ }^{6}$ possessing, controlling, or having access to resources which allow them to potentially promote their interests over the interests of others. Resources refers to money, influence, personal charisma, sporting prowess, family ties, control of the media or any other personal or external assistance capable of being used to promote the actor's interests (Clark 1968:57). Power vested in public officials is more correctly described as authority, the defining characteristic of which is its political legitimacy. Authority refers to being given administrative control of decision making processes and scarce resources on behalf of 'the public' to be administered on their behalf. I now turn to the links between power and corruption.

It is not the intention of this chapter to haggle over definitions of corruption. Most authors tend resignedly to accept the Transparency 
International definition: 'the misuse of public power for private profit'? I propose to expand it slightly.

Corruption refers to the actions of government officials, whether elected or appointed, who use power, in the form of the authority vested in them on behalf of 'the public', to promote their own interests over that of the public. ${ }^{8}$ Corruption also refers to the actions of private actors who conduct transactions of power with public officials, characterised by supplying the official with a convertible resource of power for the purpose of gaining access to government controlled resources that allow the private actor to promote their interests over that of the public. It will be noted that corruption has been the mechanism by which the authority possessed by public officials has been transformed into power that can be used to serve the private interests of both parties to the transaction. ${ }^{9}$

The key point of this definition is that it focuses on the dichotomy between public versus private interests and the link between power and goal achievement (Etzioni 1970:19). It becomes clear when you overlay the definitions of corruption and power that corruption is a technique of goal achievement, not an end in itself. Given that power is essentially a social process these definitions place corruption centrally within the ambit of all social and therefore power relations. ${ }^{10}$ We are talking about the most fundamental human characteristics: cognition; goal achievement and the use of power; and, human relations in social networks. Which brings me to the basic building block of social relations, the dyadic interaction between two actors.

\section{Social relations and corrupt social networks}

The precise nature and conditions under which social interactions take place are the subject of much debate (Parsons 1951). I will focus on only two aspects of social interaction crucial to corrupt transactions-secret communication and trust.

\section{Secret communication}

Corrupt transactions are by definition sanctionable. If they are sanctionable, whether legally or otherwise, those that participate in them know they must keep such participation secret from others. This raises the first and perhaps most difficult hurdle to overcome for actors wishing to instigate a corrupt transaction. They must open a channel of communication with the intended target of their corrupt approach.

A channel of communication must have a medium by which communications are maintained. In normal social interactions this is simple to conceptualise. One person meets another in a restaurant and has a 
conversation. They talk on the phone, send a letter or communicate by any recognised means. The opening of the channel of communication is a difficult dance for the instigator and the target. The approach can't be too obvious, lest the target denounce the instigator and expose them to sanction even before any benefit is possible. Nor can the approach be too abstruse lest the target misunderstands the true nature of the overtures being made and the opportunity is lost. The instigator doesn't want to be in situation where they have to spell out the nature of the approach to clear up misunderstandings. I would suggest, and experience bears this out, that corrupt approaches are usually made in face to face meetings where as much non verbal information can be conveyed and received in what is a highly complex social interaction. In my ten year's experience as a corruption investigator, I have never seen a first corrupt approach made by telephone or by letter. ${ }^{11}$

This is why interest groups are willing to pay huge amounts to have 'the ear' of political decisionmakers. They know that without a channel of communication there is absolutely nil prospect of influencing a decision maker's decision. Most modern political systems, which have safeguards against corruption, are still struggling to deal with the payment of large sums for 'access' to politicians. Indeed, in Australia, it is standard practice for political parties to hold policy 'forums' where interested business groups pay tens of thousands of dollars for guaranteed access to the Minister.

Once a channel of communication has been established and one corrupt transaction executed, the nature of the communication changes between the two actors. There is no longer an instigator and a target, only two willing participants. The corrupt transaction is sanctionable and secret ensuring the channel of communication cannot be closed even if one actor decides they no longer want to participate.

\section{Trust}

The two actors are now locked in to a relationship that has its own demands. At any time they could be revealed and exposed to sanction. This requires that the corrupt relationship be conducted in total secrecy and with a great degree of trust between the participants. Luhmann (1979) describes trust as a generalised expectation that the 'other' will continue to behave in accordance with the way they have portrayed themselves. In essence, trust is the rational response of actors to increasing social complexity. It is impossible to have all the information we need to make decisions about the behaviour of others and where it will lead so we make an assumption of trust. Such assumptions vastly reduce the computational complexity of decision making in a social world. As Luhmann notes, one can reduce complexity and therefore the need for trust in modern society 
by relying on structure. Trust in most commercial relationships has been to some degree displaced by complex legal and normative frameworks, which govern the way business is conducted (Luhmann 1979:34).

In corrupt transactions there is no such structure to rely on. The secret and sanctionable nature of the transaction ensures that the actors must 'trust' each other. ${ }^{12}$ Once trust is established, the actors are locked into a complex social interaction in which trust must be nurtured and maintained. Therefore, the channel of communication between the actors must be of a nature that nurtures and maintains such trust. I would argue that this is why personalised relations are so important to ongoing corrupt transactions. It is often the case that corrupt actors must meet face to face, socialise and generally enjoy some sort of empathy that engenders trust between them. Face to face personalised relations actually affect the nature of the corrupt relationship. Trust, empathy and desire to fulfil the actors' personal interests interact in complex ways.

The need to build and maintain trust through face to face relations may explain the fact that in many corrupt transactions the target will accept a resource as the bribe which is vastly lower than the 'rational market value' of the publicly owned resource they are swapping. Public officials simply sell out for a lower than expected price. This is particularly evident in police culture where police will risk their reputation, career and superannuation for a few cartons of alcohol, a free night at a brothel or a very small amount of money. The annals of the New South Wales Independent Commission Against Corruption (ICAC) are full of examples. ${ }^{13}$

\section{Corrupt networks of exchange}

Most of the theorising about corruption is based on the corrupt dyadic exchange. With a few notable exceptions very little work has been done on corrupt networks. ${ }^{14}$ Corrupt networks of exchange highlight the essentially social nature of corrupt transactions. I will now attempt to outline a model of how corrupt dyadic transactions can develop into corrupt networks of exchange. It should be noted this is a theoretical model in its infancy and much further work is needed. To assist in the understanding of corruption as a social process I draw on insights from a mode of inquiry which uses networks to understand social interactions. This approach goes under many guises but is commonly known as Social Network Theory (SNT). ${ }^{15}$ SNT focuses on actors as nodes in a network and the importance of connections between actors (Emirbayer and Goodwin 1994). SNT sets out to understand the patterning of actor's interactions. It is based on the intuitive idea that the patterns of interactions between actors in a social network have a strong 
affect on the way that actors live their lives (French 1956). This approach combined with increased computing power has led to the development of a way of visually representing social networks that allows comprehension of their complexity in terms of space and over time.

Imagine that every person in the world is a node in one gigantic network. ${ }^{16}$ Every actor has links with other actors in the network, which form complex sub networks. Some have a few links and some have many. Some actors have high quality connections through which high quality communications flow. Some have low quality connections and their communication with others will be degraded and unsatisfactory for their lifetimes. A feature of this social network is the interconnectedness of the actors who form the its nodes. Social researchers have been continually surprised by the interconnectedness between actors, even in a huge and complex society like the United States. ${ }^{17}$

This system is not static but dynamic. Connections are constantly growing, connecting, and dying. These social connections are modified by the experiences of each individual actor. Social connections require energy investment to be maintained. If individual actors do not invest in each connection then eventually they will atrophy. ${ }^{18}$ Unlike biological neural networks, social networks do not have a central organising principle, in response to stimuli external to the network. Actors in social networks grow connections according to their own needs, desires, environmental and social influences. They respond to the connections grown toward them in a similar manner.

Typically, for each actor, they have strong, smooth, wide and well-serviced connections with their immediate family, mother, father, partner, siblings and children. ${ }^{19}$ They will have connections of a different character with friends and acquaintances. Connections with family will generally stay constant throughout life. Connections with friends and acquaintances will grow and die in accordance with the particulars of each relationship. Each actor will have connections with their employer and other employees in the workplace. They may have a formal hierarchical relationship with the boss, but formal and informal connections with fellow employees (Argyle 1988:174). Actors may have connections with other actors who share their interests. ${ }^{20}$ They may belong to a political party or a gardening club or be the local scoutmaster. They may go to a dance group on Thursdays and Church on Sundays. Everywhere they connect with other actors thus increasing the complexity of their relationships and their place within networks (Brown 1965:160).

Generally the connections actors make are the result of circumstance and intention. ${ }^{21}$ Actors join or become part of definable groups or less 
definable networks. ${ }^{22}$ Initially their main connection with others in such groups is the common interest of the group. As connections grow and mature within the group, this changes over time, although the group interest will always be in the background providing an underlying structure for the group connections.

Actors are intentional and can form connections in directions that suit their needs and desires. Actors do this to varying degrees and fall on a continuum between being passive and shaped by the forces acting on them, to actively forging connections strongly in the direction they want to go (Fischer 1977b). The question then becomes what are their needs and desires and how much capacity does each actor have to achieve them?

The precise mechanism of needs/desires/goal formation need not concern us here. Humans often act irrationally out of fear, greed, envy, jealousy or just plain stupidity. They do not act the same way all the time, in all situations. The complexity of human action falls within the realm of psychology the most that can be said is that humans act in accordance with their perceived interest at that particular time and place. ${ }^{23}$ Once needs/ goals/interests have been established in the mind of the actor they must then be sufficiently motivated to act.

I have described a social network where every actor inhabits a location within the network connected to others. The connections are growing and changing and the actor can intentionally create connections to assist in achieving their goals. Actors can only achieve their goals if they have possession, control or access to resources that allow them to do so (Burt 1977:269; see also Etzioni 1970:19). In the context of a social network, the possession, control or access to such resources gives the actor 'power'. In modern economies the most commonly possessed and used resource of power is money, however anything at all can be a resource of power if it assists an actor to promote their interest over the interests of others. Charisma, public speaking ability, media resources, advertising, and information that others don't have can all be resources of power (Olsen 1970:5).

Gathering resources is the way an actor gathers power. After all, power is a potential state, not an actual one. When an actor uses power and actually achieves their interest over the interest of someone else then power has been used. If the actor has the ability to promote their interest over another but they haven't actually done so then they have power but they haven't used it. ${ }^{24}$ Emerson goes one step further and suggests that power is not an attribute of a particular actor per se, but of the social relations between actors. If an actor controls resources that another actor is dependent on, then to that extent the first actor has 'power'. 
Every actor controls resources that they can call upon to promote their interest. They also possess or control resources that can't be used to promote their interest. Money is such a useful resource of power because of its convertibility. It can be handed from one actor to another and be immediately converted to the use of the new owner. This analysis appears to lead to corrupt transactions being a straightforward market in resources. Rose-Ackerman is right to point to the allocation of scarce government controlled resources leading to the creation of a market, as being central to understanding corrupt conduct (Rose-Ackerman 1978:2). What RoseAckerman and most economists fail to comprehend is that the market is merely a simplified representation of complex networks of social exchange and social action. The general population is so used to participating in the buying and selling of material goods that the layers of social exchange that underpin such transactions are invisible and instinctive (Jackson, Fischer and Jones 1977:43). Social exchange theorists propose that social exchange occurs between connected actors in relation to all transacted resources, whether material or non material. Such transactions are based on dependency relations, which lead to the operation of power (Cook 1987).

The above discussion brings us to the dyadic corruption transaction which always includes an instigator and a target. Once the instigator has made the decision to make a corrupt approach they will attempt to contact the target and persuade them to comply. It will be obvious from the above discussion that this attempt at convincing another individual to be part of a corrupt transaction involves power from both the instigator's and target's point of view. This attempt at a corrupt transaction will either succeed or fail-there is no in between state. It can only have a chance of succeeding if the following conditions are met

- The instigator has a channel of connection with the target (Dahl 1994:292).

- The instigator has a resource the target wants or needs.

- The target is dependent on this resource.

- The target cannot easily obtain this resource another way.

- The target has a resource the instigator wants or needs.

- The instigator cannot easily obtain this resource another way.

- The connection between the instigator and the target is of sufficient quality that allows 'trust' between the actors to support the transaction (Della Porta and Pissorno 1996:76).

- The transaction can be conducted in secret (Alatas 1968).

- The target will have no moral objection. ${ }^{25}$

- The target perceives that the risk of sanction and the 
consequences of sanction are low enough to allow the transaction.

- The instigator perceives that the risk of sanction and the consequences of sanction are low enough to allow the transaction.

Whether the instigator makes the attempt at a corrupt transaction at all will be contingent on a combination of the above factors accumulating a sufficient 'weight'. The acceptance of the corruption attempt by the target will also be contingent on the above factors accumulating a sufficient weight to allow receipt of the attempt. It is clear from the above discussion that any corruption/power attempt (CPA) involves excitatory factors that motivate the attempt and inhibitory factors that prevent it.

If the CPA is successful then two consequences ensue. First, the connection between the actors is strengthened. Second the channel along which such CPAs occur will remain open. All the above conditions having been met, the instigator merely has to provide a resource that the target desires to continue such transactions. In addition the instigator has the power resource of information regarding the target's previous corrupt transaction. ${ }^{26}$ Gaining evidence of the original or subsequent transactions strengthens this. If the target is dependent on preservation of her good name, as all public officials are, then this resource further adds to the instigator's power in the relationship. This connection is now ready to be used in continuing corrupt transactions.

For actors in the corrupt network the connection itself will have to be maintained and both actors in any transaction (either a power attempt or a corrupt power attempt) will have to expend resources to preserve the secrecy of their transactions. The two actors are now tied together in such a way that continuing trust is required. ${ }^{27}$ Although the transaction began with an instigator and a target, future transactions will be characterised by their mutuality. Further improvement of the connection by personalising relations via social means may take place. ${ }^{28}$

The instigator in a corrupt transaction can be either a government actor or a private actor. I would suggest that if a government actor has high motivational forces and low inhibitory forces then they will probably be the instigator. This is not usually the case in modern democracies where the rule of law is established and enforced. The rule of law and widespread education about the unacceptable nature of bribery ensures that moral inhibition, perceived risk and consequences of sanction are generally higher than in jurisdictions where the rule of law is not well established or enforced, such as in developing countries. In addition, public officials in developing countries, due to their poverty, are highly resource dependent 
on material goods to just survive therefore ensuring they have high levels of motivation..$^{29}$

If the instigator's desire for resources to increase his power has been satisfied then their relationship may remain static. A non-government instigator may attempt further corruption attempts against other government actors, through the target, if the instigator has a desire for power that exceeds the ability of the target to satisfy. Corruption will tend to flow upwards through the authority hierarchy to the next level of management, in order to gain access to greater discretional authority. This is particularly significant in democracies and modern bureaucracies where authority is usually scattered among a number of actors. It is not usual for one actor to possess monopoly decision making authority over significant government controlled resources. ${ }^{30}$ An instigator may attempt to corrupt other public officials if other actors in the instigator's own network wish to access the instigator's connection to further their desires for resources of power.

The key point is that the instigator (who we will assume is a nongovernment actor) is unlikely to have a readymade connection with potentially corrupt government actors who can provide government resources that she desires. Having gained access to one corrupt government actor the instigator now has the ability to connect with all the government actors linked to the original corrupt actor. Through one connection there is potential for corruption to pervade the entire network. The process of opening up the network to corruption can repeat itself for as long as: there are targets in the government agency amenable to such an approach; the agency has resources of power desired by the instigator(s); and the instigator(s) have resources that can be converted to the use of the targets. In addition, any attempt has to satisfy the conditions specified above. This however is complicated by the fact that as the network grows, corrupt network actors are in the unique position of being able to use organisational power to negate some of the inhibiting conditions.

It should be noted that prior to the establishment of a corrupt network there is no overriding organising principle patterning the actor's relations with the other potentially corrupt actors, with the exception of the preexisting organisational structure and informal social influences. Once the corrupt network has been established then it is subject to dual influences. Firstly the corrupt network is subject to the personal agendas of the actors, fuelled by the relative power of each actor in the network. Secondly the corrupt network is subject to the overriding influence of the whole corrupt network. The consequences of this is that actors in the network will fire power attempts and corrupt/power attempts in directions that promote the network as well as specific acts of corruption. 
The result will be a continual firing of power attempts between actors in the corrupt network, and between actors in the corrupt network and other organisational actors. Organisational power, power attempts and corrupt/power attempts, will be used by corrupt network members to grow the network toward places in the organisation where discretionary power resides and can be utilised to the network's advantage. This will include the promotion of network members and the isolation and ejection of nonnetwork members. Individual actors will interact with this process in a complex manner, patterning the way the network grows.

Internal motivating forces will drive or inhibit the corrupt actor's corrupt behaviour combined with external organisational factors such as low morale, poor leadership, lack of moral leadership, poor training, ambiguous organisational ethics and impractical policies and procedures which act to corrode moral inhibition. ${ }^{31}$ Other factors such as poor accountability, internal audit review, performance management and supervision can lead to a perception of low risk of sanction and reduced consequences of sanction.

Once a corrupt network of actors is established in an organisation then the use of power is expanded through resource transferral. The corrupt actors maintain and protect themselves and the network through the use power in all its forms. ${ }^{32}$ Participating in a corrupt network places the actors within a group that possesses a collective interest-that of furthering corrupt transactions and protecting the network.

Members of the corrupt network do not have to go outside the network to gain power in the organisational context. They all have access to organisational power to varying degrees, however the collective nature of this power means that as the network grows the organisational power available to its members grows exponentially. At a certain point the accumulation of organisational power reaches a critical mass and at this point the corrupt network has captured the organisation. It should be noted that the power of the corrupt network does not relate necessarily to the number of actors involved but the scale of organisational power available to the corrupt actors in the network. ${ }^{33} \mathrm{~A}$ few corrupt actors with a high degree of organisational power can capture an organisation as can a large number of corrupt actors with a smaller amount of organisational power. ${ }^{34}$

\section{Corruption networks and corruption prevention}

How does understanding corruption in terms of social interactions and social networks assist in preventing it? Firstly it provides a theoretical basis for the value of the organisational integrity approach. As Boardman and Klum note in this volume, 'unethical behaviour' is causally linked to a number of risk factors. Through experience and some descriptive research 
it has become apparent that corruption can flourish where certain risk factors are present. An examination of the literature suggests that at present there is no theoretical understanding of the functioning of corruption which explains how these risk factors lead to corruption.

The organisational approach can be successful because the culture of public sector agencies is to some extent controllable. Through education, corruption prevention and the promotion of corruption 'resistance' a powerful atmosphere of organisational integrity can be created. Such an atmosphere can contribute significantly to an actor's inhibitory motivational forces. The approach does however have its limitations as it fails to appreciate the other side of the equation - the excitatory factors that impel the individual to action. If such factors outweigh the inhibitory forces created by the organisational integrity measures, then corruption will still occur.

Two examples will illustrate my point.

1 A public official in a developing country could be still be strongly impelled to act corruptly, even if her agency has implemented successful organisational integrity measures, if her family is starving and corrupt transactions will feed them.

2 In the United States of America narcotics interdiction officials are highly trained, well paid, and subject to strict codes of conduct, oversight, auditing and other measures. Corruption is still a problem partially because of the massive sums available to narcotics traffickers to bribe officials. Those that can't be bribed can be intimidated through threats to their families.

It is not true to say that everyone has their price. It is however true to say that there will always be some individuals for whom the impulsion to act corruptly under certain circumstances will be stronger than the inhibitory forces resident in the institution to which they belong. The above examples highlight the importance of the effect of resource dependency on human action. Corruption prevention programs should strategically target resources available to corrupt private actors as well potential resource dependencies for government actors. Understanding resource dependency could inform programs of risk assessment targeted at vulnerable officials.

The social networks approach alerts us to the importance of channels of communication. Without a channel of communication a corrupt transaction is impossible. Corruption prevention programs should include the monitoring of channels of communication with potential corrupters. Payment for access to public officials should be seen for what it is-an attempt to open a channel of communication that allows influence.

Once a channel of communication is opened between two actors in the network which have conducted one corrupt transaction, it remains open, 
even if the actor in the public sector agency moves to another position. Public sector officials suspected of corruption cannot be just moved or 'promoted out' as so often happens with problem employees. Those suspected of corruption must be dealt with by the agency through the disciplinary process. If sufficient evidence is available strong steps should be taken to remove them. If this is not possible then they should be the subject of overt or covert surveillance to affect and monitor the suspect officer's behaviour.

The social networks approach to corruption focuses on the way power is an integral part of the functioning of corrupt networks, both in terms of the gathering of resources for goal achievement, and as a medium of exchange in the network itself. Corruption is a technique used by power holders and power seekers to gain or maintain power. Corruption is not an end in itself, merely a technique of goal achievement and corruption is but one technique of gathering the power needed to achieve such goals. Actors willing to use corruption to achieve their goals are also willing to use more subtle techniques to achieve their ends. In particular, this approach suggests that the misuse of organisational power, even though it might not appear to be for private gain, is a clear indicator of the potential for corruption in an organisation. In corrupt networks, operationalising the network can, at certain stages, be a lot more important than the exchange of resources. The use of organisational power to facilitate nepotism may be of much more significance than traditional examples of corruption. Once a corrupt network has been operationalised in key areas of the organisation, the chance for discovery and change have been vastly reduced.

Focussing on the interests of actors in corrupt networks suggests that the shared interests of actors in corrupt networks is a key issue. Any corruption prevention program should target the potential for strongly shared interests of public sector employees where such shared interests are not identical to that of the organisation. As we have seen, trust is a key component of relations between actors when the first corruption attempt is made and in ongoing corrupt relationships. Shared interests between actors facilitate trust and provide a common motivational stimulus that can override the inhibitory forces that may be active.

Shared interests can provide a readymade network that contains its own culture, norms and goals, which can displace or negate organisational cultural norms. The most obvious example in Australian political life is the strong culture of political parties. This can lead to, and has in many cases, the corrupt elevation of party political shared interests over the democratic institutions of parliament, which the members of the party are supposed to serve. Any corruption prevention program would do well to identify shared 
interests of power holders in public sector employment and to focus on these as potential nodes of corrupt influence.

Finally, and perhaps most importantly, the social networks approach to corruption allows the corruption investigator to conceptualise the operation of corrupt networks in terms of power flows and relationships rather than the attributes of actors. The question to be asked by the corruption investigator is not whether actor A is corrupt. The question should be, what is the relationship between actor $\mathrm{A}$ and other potentially corrupt actors in the network. Understanding corrupt networks in this way allows us to comprehend that corrupt transactions can occur across the network between individuals who are not directly linked. Such transactions, on their face may not appear to be reciprocal and may be removed in space and time. It is only by understanding the relationships between the actors in the network that the true nature of such transactions can be revealed.

\section{Conclusion}

The problem for corruption prevention programs is that corrupt networks and social networks look similar to each other and function in the same way. We all operate in a world of differentiated social networks based on communication, personalised relations and shared interests. To act socially is a human need. It is simply not realistic to believe that rational managerialism can prevent the creation of social networks involving public sector agency officials. To radically restrict personalised human interaction would reduce efficiency in public sector agencies to such a low level as to make them non viable. Weber's totally rational bureaucracy is an idealised fiction that is not possible and not desirable. The challenge for anti corruption agencies is to attempt to formulate a model of bureaucracy and policy formulation that can integrate the reality of personalised relations and social networks whilst ensuring decisions are always made in the interests of the 'the public' alone.

\section{Notes}

${ }^{1}$ For the sake of brevity I will condense these concepts into the word 'interests'.

${ }^{2}$ This is a relatively uncontroversial view in modern psychology. See for example Lamberth (1980:295) and Atkinson, Atkinson and Hilgard (1983:317).

${ }^{3}$ I am utilising concepts of power dependence relations derived originally from social exchange theorist Richard Emerson. See for example Emerson (1962).

${ }^{4}$ Weber defined power as the ability to achieve one's goals despite opposition from others in a social relationship (quoted in Giddens (1971:156)).

${ }^{5}$ Emerson wrote, "The dependence of actor A upon actor B is (1) directly proportional to A's motivational investment in goals mediated by B and (2) inversely 
proportional to the availability to those to A outside of the A-B relation' (1970:45). In essence, he is saying that $A$ has power over $B$ to the extent that $B$ is dependent on resources controlled by A.

${ }^{6}$ Which can be an individual or an organisation

${ }^{7}$ Originally proposed by Joseph Senturia, 'Political Corruption', Encyclopaedia of the Social Sciences, Vol iv: p448.

${ }^{8}$ One problem with this definition is that the public official may rationalise that they have used their power in a way that benefits both themselves and the public interest. In this case they have promoted their interest in equal proportion to the public interest rather than over it. My view is that they intend to promote their interest regardless of the public therefore the fact that these interests may coincide is irrelevant. In addition, by promoting their interest they have distorted the process of achieving the 'best' policy, which in itself is against the public interest.

${ }^{9}$ It will also be noted that this definition faces the difficult problems of how you identify the private interests of the public official/private actor and how you identify the 'public' or 'common' interests of the populace? These are issues I don't intend to address here.

${ }^{10}$ It is almost self-evident that power can only be exercised in relation to others. See for example Bierstadt (1994).

${ }^{11}$ An extraordinary example of this has recently occurred in Peru where it is estimated that 2400 video tapes were made of corrupt approaches between the head of Peru's secret police, Vladmiros Montesinos and politicians. All of the corrupt approaches occurred at videotaped meetings in a hotel room. See Associated Press (2001).

${ }^{12}$ It should be noted that one off corrupt transactions which have no prospect of being repeated, such as the license providing official who extorts a 'fee' from the hapless applicant, have a somewhat different nature and are not included in the current discussion.

${ }^{13}$ Perhaps the best example is an investigation conducted into the unauthorised release of government information. Police were risking their careers for $\$ 10-20$ per item of information even knowing their computer use could be audited. Independent Commission Against Corruption (1992).

${ }^{14}$ See Cartier-Bresson (1997) for one of the most thoughtful and interesting discussion on corrupt networks.

15 'Social network analysis is not a formal or unitary 'theory' but rather a broad strategy for investigating social structure' (White and Brudner 1996). See also Leinhardt (1977). SNT also goes under the name of network analysis (Fischer 1977a).

${ }^{16}$ The concept of each individual as an actor enmeshed in a social network links to early work by sociologist George Simmel in 1922 (Fischer 1977b).

${ }^{17}$ See for example Travers and Milgram (1969). They conducted an experiment to see how many people through personal acquaintances could transmit a letter from the West Coast of the USA to the East Coast. Approximately 30 per cent of the sample letters made it to the East Coast, travelling through a mean number of acquaintances of 5.2. This was the basis for the so-called 6 degrees of separation where every person on the planet is only six acquaintances from knowing every other person. Obviously overstated, Milgram's research does illustrate how amazingly connected even complex modern societies are. 
${ }^{18} \mathrm{I}$ am referring here to the importance of maintenance of interaction and the motivation to behave appropriately using social skills (Argyle 1988:52-56).

${ }^{19}$ Although within this network there will be considerable variation (Bott 1977).

${ }^{20}$ There has been considerable work done on why people form relationships, apart from structural or environmental factors. The research suggests that one important factor is 'solidarity' or having similarity in characteristics and interests (Brown 1965)

${ }^{21}$ Theoretical approaches to this question vary along a continuum. The ratio of the effect of environment vs intention (and therefore formation of connection) is very much in dispute (see Fischer 1977b:3).

${ }^{22}$ The difference between a network and a group is difficult to establish. Generally in the social sciences a group has members that have larger common aims, 'interdependent roles, and a distinguishable subculture'. In networks, only some of the actors have social relationships and they don't form a recognisable definable social whole (see Bott 1977).

${ }^{23}$ This approach is in line with the more sophisticated versions of rationality outlined in the Social Choice literature, or as Jackson et al. (1977:42) call it, 'structured choice'.

${ }^{24}$ Defining power, potential power and influence is surprisingly controversial. For discussion of this debate see Martin (1994), Clegg (1979:41-49), Molm (1987:101).

${ }^{25}$ Sometimes referred to in the economics literature as the 'moral cost' (see Groenendijk 1997).

${ }^{26}$ Information is clearly an important resource of power. In corrupt transactions it becomes crucial (Garson. 1977; Olsen 1970).

${ }^{27}$ Della Porta found, in her massive study of Italian corruption, that 'honesty' was a valuable trait among corrupt actors. If you were generally known to be 'honest' in your corrupt dealings then you were a person that could be dealt with (Della Porta 1996).

${ }^{28}$ In the author's experience as a corruption investigator with the NSW ICAC, this is a particularly common phenomenon. Della Porta (1996) also found that personalised relations were an important factor in ongoing corrupt transactions.

${ }^{29}$ It is axiomatic that low pay is one of the key causes of corruption in developing countries (see Palmer. 1983; Alatas 1990).

${ }^{30}$ Disagreement exists between the élitists and pluralists about how centralised decision making authority is. In Australian government, élites do exist and have significant power however it is mitigated (Higley, Deacon and Smart 1979). As RoseAckerman points out, in modern democracies the power of élites is mostly to block or deny others, rather than to act or approve (1996:377).

${ }^{31}$ The effect of such factors in potentially promoting corruption is brought out in Zipparo (1998).

${ }^{32}$ Martens (1999) provides practical detail as to how this occurs.

${ }^{33}$ Warren (1977) notes that formal positions in the organisation may not relate to actual power structures.

${ }^{34}$ By organisational power, I am not referring to the resources available to the organisation. I am referring to the ability of the actor to influence the workings of the organisation itself. 